\title{
Femoroacetabular impingement inducing non-union of a femoral neck fracture: a case report
}

\author{
C. G. Lenz $\cdot$ P. O. Zingg $\cdot$ A. F. Kamath • \\ C. Dora
}

Received: 7 August 2014/Published online: 6 March 2015

(c) Springer-Verlag Berlin Heidelberg 2015

\begin{abstract}
Introduction We describe a case in which femoroacetabular impingement (FAI) was identified as the cause of non-union of a femoral neck fracture and the subsequent treatment strategy.

Materials and methods Retrospective review of a 35-year-old patient, without any risk factors for non-union, who sustained a femoral neck fracture. Pre-existing FAI was identified as the cause for the non-union of the femoral neck fracture, with successful treatment of the non-union according to established arthroscopic treatment of the hip. Results After treatment of the FAI, the non-union healed uneventfully within 3 months.

Conclusions FAI may be a less common but potential cause of delayed union or non-union in the setting of femoral neck fracture in the young.
\end{abstract}

Keywords Femoroacetabular impingement (FAI) ·

Femoral neck fracture · Non-union · Arthroscopy ·

Osteochondroplasty

\section{Introduction}

Displaced femoral neck fractures in young adults necessitate anatomic reduction and stable internal fixation to preserve the femoral head, achieve union, and avoid

C. G. Lenz $(\bowtie) \cdot$ P. O. Zingg · C. Dora

Orthopaedic Department, Balgrist Hospital, University

of Zurich, Forchstrasse 340, 8008 Zurich, Switzerland

e-mail: christopher.lenz@balgrist.ch

A. F. Kamath

Department of Orthopaedic Surgery, University of Pennsylvania,

Philadelphia, PA, USA osteonecrosis. Non-union rates as high as 10-30 \% [1-4] have been reported and associated with either general risk factors such as systemic disease, alcohol and other drug abuses, and/or local vascular, infectious, and mechanical factors. Inappropriate fracture reductions [5-8] such as varus angulation and/or posterior tilt $[5,7,9,10]$ increase non-union rates up to $50 \%$ [5]. Barnes et al. [5] also showed differences regarding the fixation method: a SmithPetersen nailing exhibited a higher rate of non-union than cross-screw fixation or a sliding nail plate (50 vs. $70 \%$ rate of union).

The role of femoroacetabular impingement (FAI) in retarding or preventing fracture healing about the femoral head and neck is poorly understood. While the dynamic conflicts between femur and acetabulum may theoretically interfere with femoral neck fracture union, this has not been described in the literature previously. We present a case in which FAI most likely caused non-union of a wellreduced and well-fixed femoral neck fracture in a healthy young man with no known risk factors for poor fracture consolidation.

\section{Case study}

A 35-year-old healthy office administrator sustained an isolated displaced mid-cervical fracture of the femur (Fig. 1) in a motorcycle accident. Of note, the patient did not recall groin pain or decreased internal rotation prior to the injury. He was treated on the day of injury by closed reduction and internal fixation with three $7.3-\mathrm{mm}$ cannulated lag screws under general anesthesia (Fig. 2a and b). Partial weight bearing was started the day after surgery and recommended for 12 weeks post-operatively. The patient was discharged home on post-operative day 4 after an 
Fig. 1 Radiograph showing a mid-cervical fracture of the left femur obtained on the day of injury
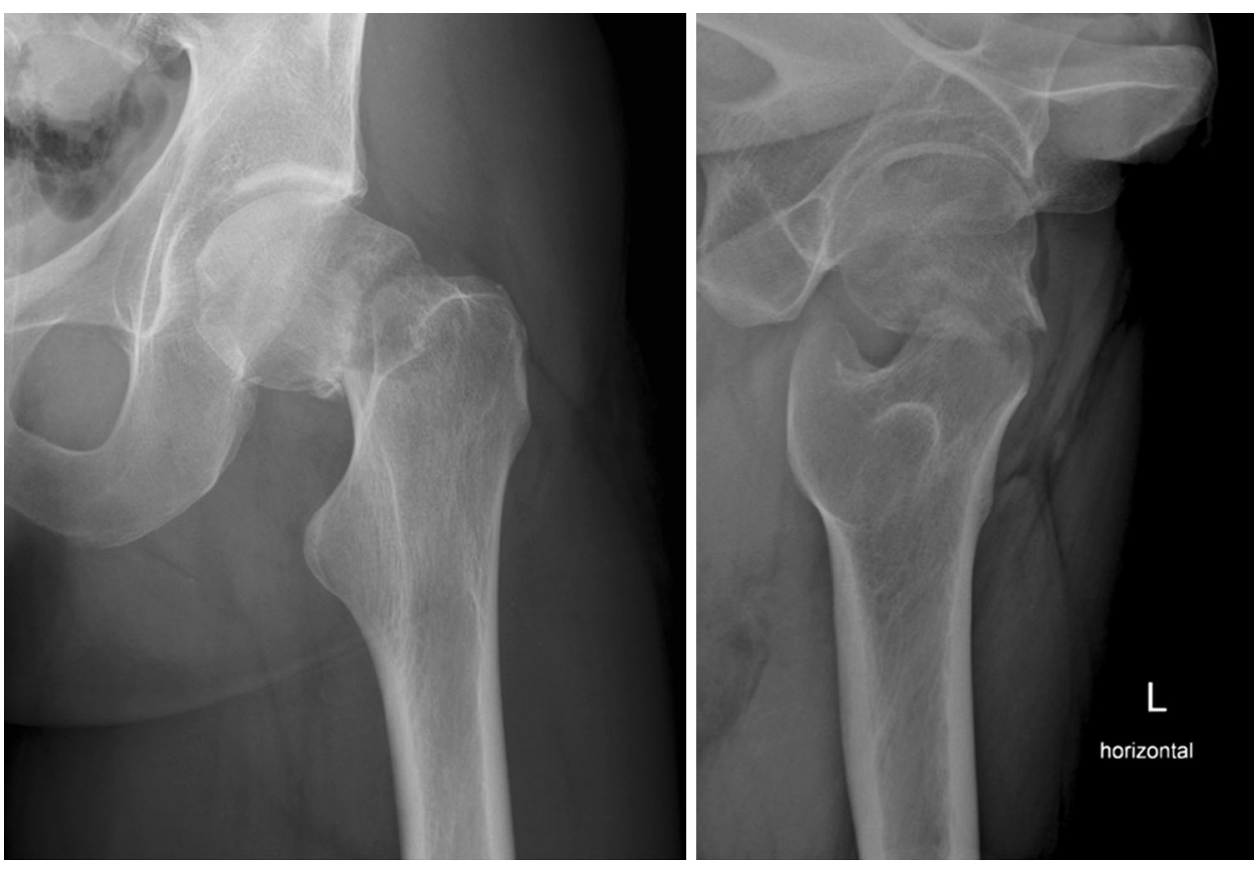

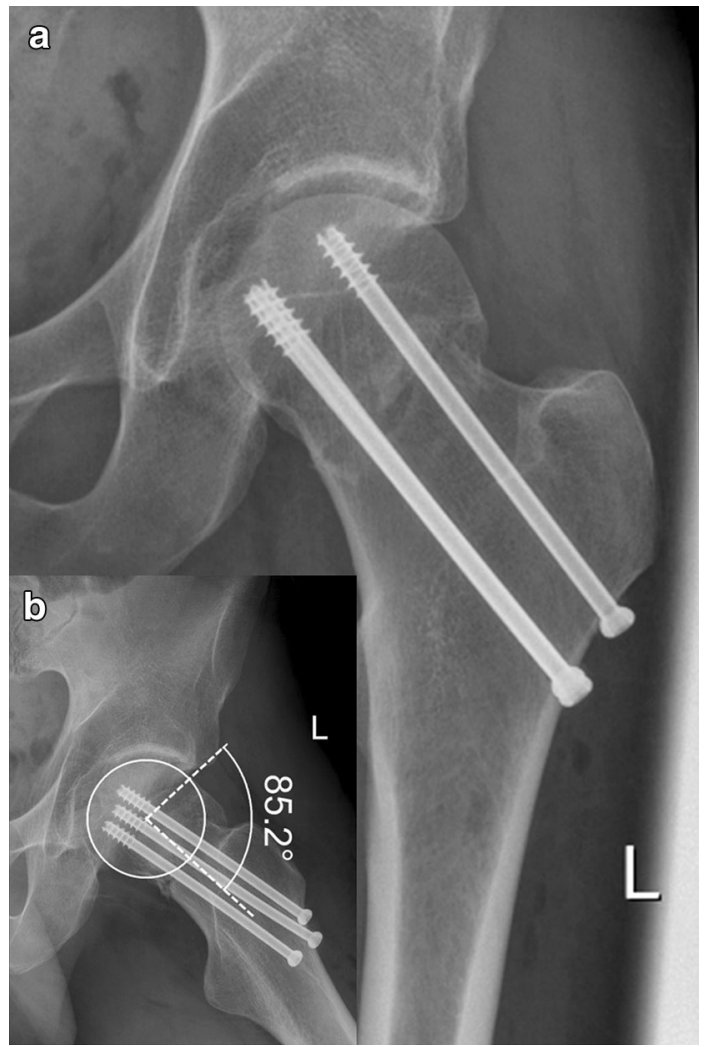

Fig. 2 Radiograph after closed reduction and screwing of the proximal femur was performed showing an adequate reduction, correct length and positioning of the screws, and sufficient stability of the fixation construct. The calculated alpha angle has been provided (b)

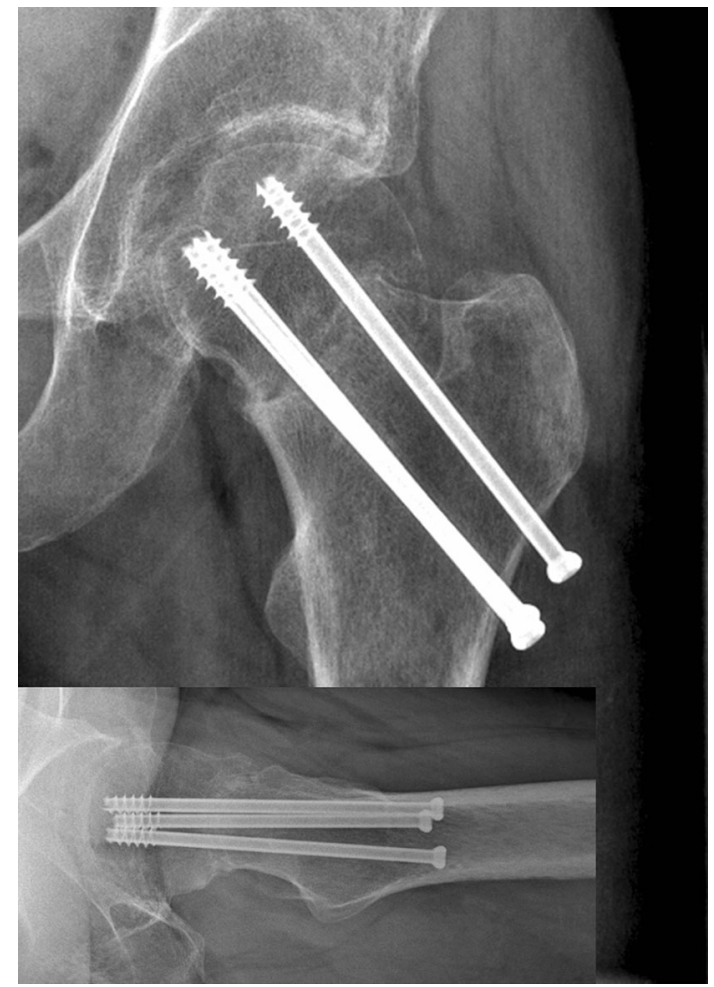

Fig. 3 Radiograph 6 months after surgery showing non-union of the femoral neck fracture

uneventful hospital course. After 12 weeks, the patient was still on crutches and not able to increase weight bearing due to persistent groin pain. 
Fig. 4 a Computed tomography scan 6 months after surgery confirming that appropriate signs of fracture union were absent; note is made of severe pre-injury cam deformity of the proximal femur. b Bone scintigraphy showing increased uptake at the fracture gap, consistent with non-union
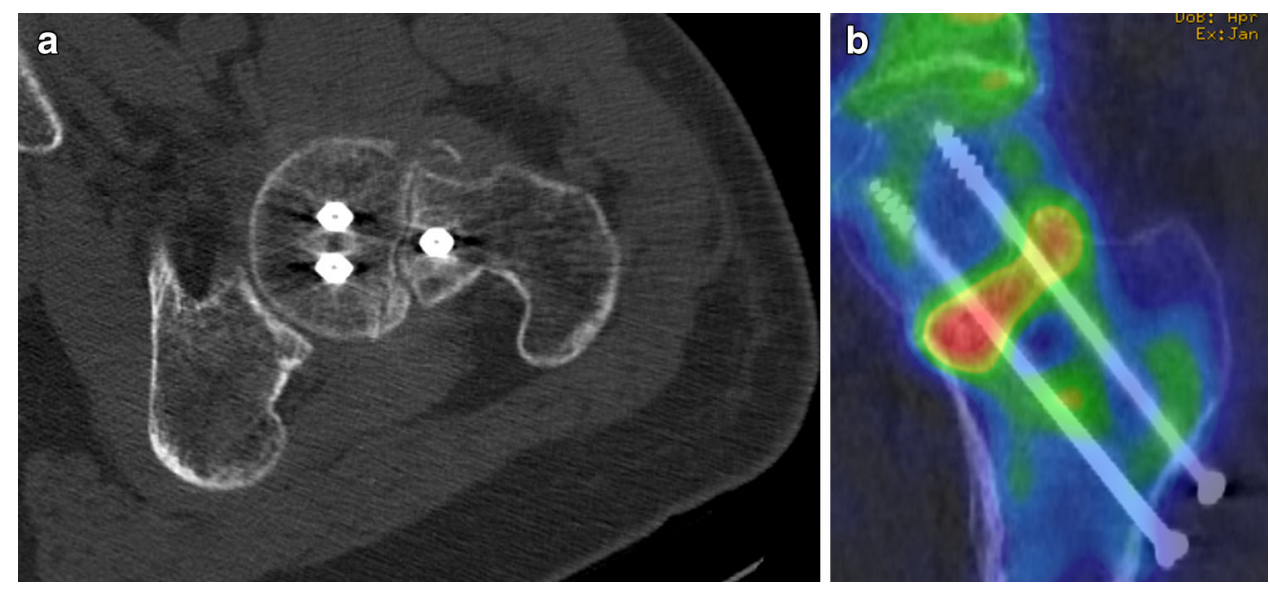

Six months after the index surgery, the patient consulted our outpatient clinic. He still endorsed groin pain during each step, when trying to bear weight, and when sitting for several minutes. Physical examination revealed a body height of $191 \mathrm{~cm}$ and weight of $86 \mathrm{~kg}$ (body mass index of $24 \mathrm{~kg} / \mathrm{m}^{2}$ ). In the supine position, the patient experienced his typical groin pain with passive hip flexion to $80^{\circ}$, as well as with $10^{\circ}$ of internal rotation at $80^{\circ}$ of hip flexion. On radiographic analysis at this visit, the fracture line was still visible, and the neck slightly shortened when compared with prior X-rays (Fig. 3). A fracture gap was seen on computed tomography scan, with unaltered reduction and no overt signs of screw loosening or hardware compromise (Fig. 4a). Appropriate shortening of the neck along the partially threaded screw paths was seen. Additionally, a pre-existing severe cam deformity was seen. On the pelvic AP radiograph, a cranial crossover sign was seen, suggesting combined cam- and pincer-type impingement.

Deep sepsis was excluded by normal white blood cell count, erythrocyte sedimentation rate, and C-reactive protein. A bony scintigram with single photon emission computed tomography (SPECT) did not reveal signs of avascular head necrosis; however, the study did demonstrate increased uptake at the fracture gap, consistent with non-union (Fig. 4b). Since there was no clear explanation for non-union, we hypothesized that a combined cam-/ pincer-impingement contributed to the fracture non-union, due to mechanical conflict and resulting micro/macro-instability environment about the fracture gap.

Because the overall fracture alignment and stability of fixation appeared acceptable, arthroscopic femoral neck osteochondroplasty was proposed and performed without complication. Patient positioning and portal placement were performed according to the technique described by Byrd [11] and under general anesthesia. In the first step of the procedure, arthroscopy of the central compartment was performed under traction. A partially ossified labrum and antero-superior chondropathy was seen. To treat this pincer component, the labrum was addressed along with acetabular rim trimming. Subsequently, femoral osteochondroplasty was performed, without traction, along the anterolateral femoral neck, taking care not to compromise the postero-superior retinacular vessels (Fig. 5). The fracture gap itself was not clearly visible, and there was no specific debridement or resection of tissue at the fracture site. Bony resection was judged adequate when, by dynamic examination and under direct visualization, an impingement-free range-of-motion was achieved, including in the position of internal rotation of at least $30^{\circ}$ with the hip flexed to $90^{\circ}$. Post-operative rehabilitation directed the patient to weight-bear as tolerated on two crutches; the patient self-administered passive hip motion on a bicycle ergometer for 6 weeks post-operatively. Indomethacin $75 \mathrm{mg}$ orally and enoxaparin $40 \mathrm{mg}$ intra-muscular were prescribed on a daily basis for a duration of 2 weeks to prevent heterotopic ossification and thromboembolic complication, respectively.

Six weeks after surgery, the patient was able to bear weight without pain, and the fracture non-union showed evidence of progressive healing (Fig. 6). At 3 months post-

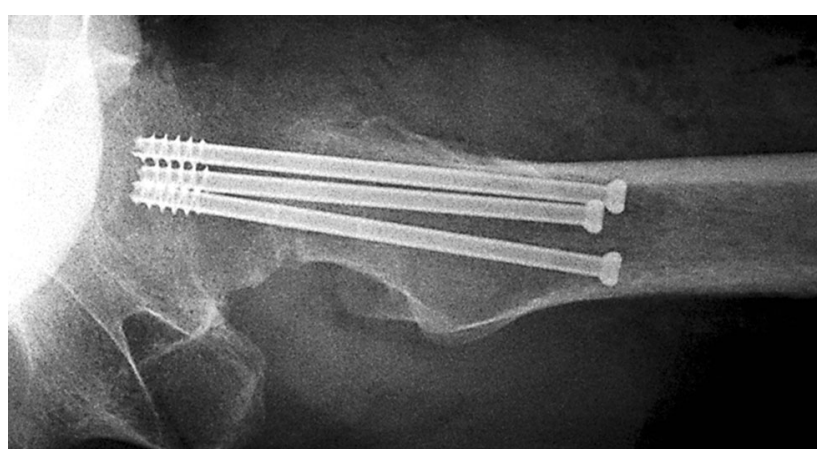

Fig. 5 Axial view radiograph after arthroscopic osteochondroplasty of the head and neck of the proximal femur with a resulting improved head-neck offset 
Fig. 6 Antero-posterior and axial view radiographs 6 weeks after arthroscopic surgery showing signs of progressive fracture healing
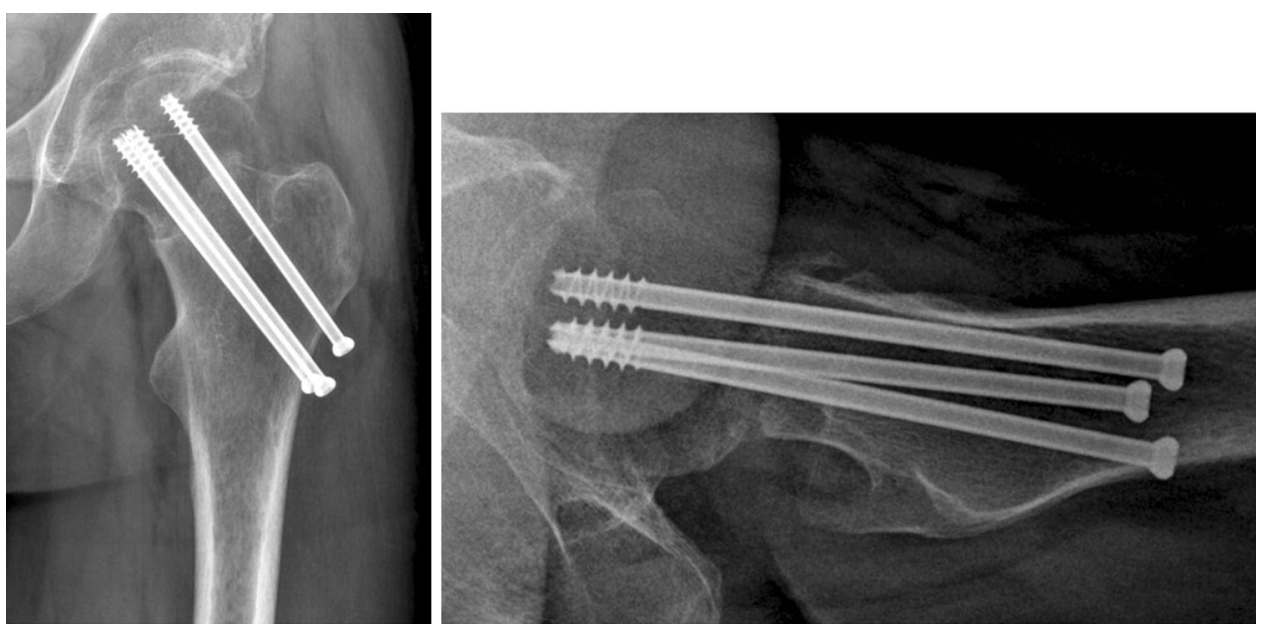

Fig. 7 Antero-posterior and axial view radiographs 12 weeks after surgery demonstrating union of the fracture
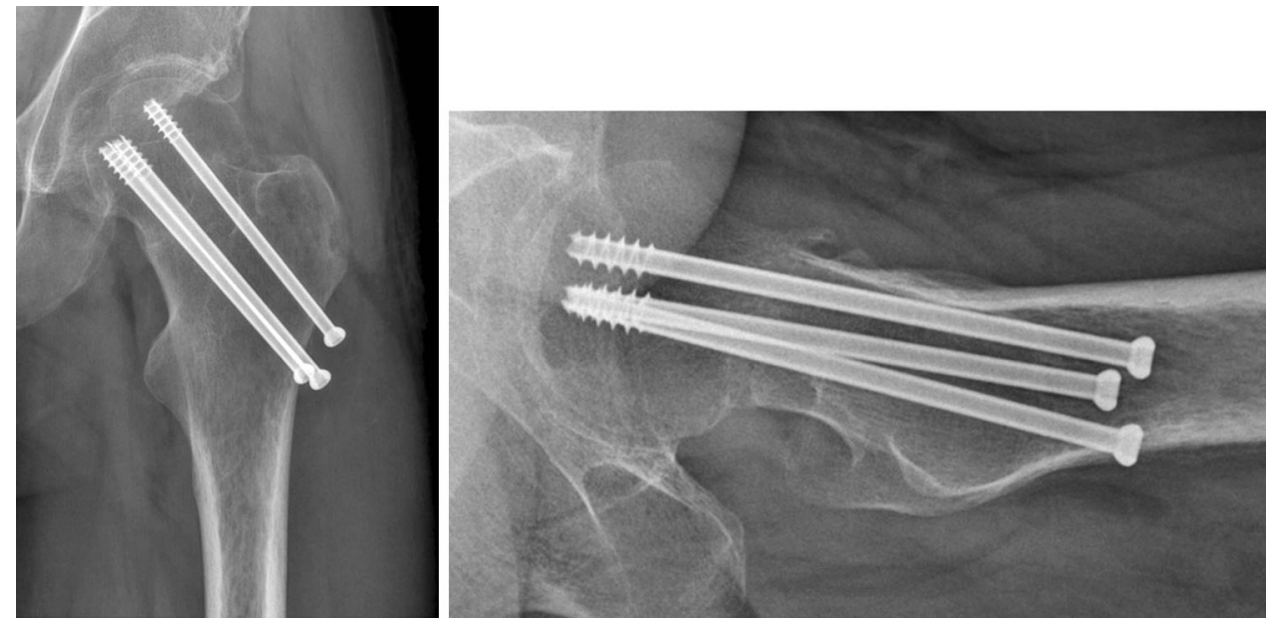

arthroscopy, the non-union had healed (Fig. 7), and the patient returned to his pre-injury level of activity and work.

\section{Discussion}

Femoroacetabular impingement triggering femoral neck non-union by levering of the fracture site when hip joint movements are reduced beyond a physiological range of motion was suggested by Beck et al. [12] in a series of 3 patients in 2004. In one case, a bony spur causing posterior impingement was removed, and in two cases, a cam-induced FAI was treated with open osteochondroplasty. However, since surgical revision included open reduction and fixation of the fracture site using a blade plate, it was unclear from this report whether improved joint clearance alone-rather than improved fracture fixation, or the two procedures in combination-contributed to union.

In the present case, arthroscopic osteochondroplasty and trimming of the acetabular rim in a combined cam- and pincer-type FAI alone rapidly resulted in fracture union and strongly highlight FAI as a major contributing factor for non-union. Absence of evidence of hardware loosening and a technically appropriate primary fixation of the fracture justified retention of the index fixation. Possibly, there is an even greater impact of the pincer component since the prominent rim leads to a direct force. During range of motion of the hip joint, the femoral neck has a direct abutment on the acetabular rim and the labrum; thus, there is a posteriorly oriented force directly onto the femoral neck. The posterior force can cause contrecoup injury adjacent to the fovea, which was not clearly visible in this case. The injury to the antero-superior acetabular cartilage is due to the cam-type component of this combined FAI.

We conclude that the presence of FAI morphologies might be a contributor to delayed union of otherwise wellreduced femoral neck fractures. The bony morphology of the head-neck junction of young patients sustaining femoral neck fractures has not been described in large groups. Therefore, radiographic and/or clinical factors 
related to either risk of fracture or response to fracture treatment and ultimate healing should be further explored with respect to FAI. Provided the fracture is well reduced and appropriately fixed, arthroscopic osteochondroplasty and trimming of the acetabular rim can effectively treat delayed and/or non-union of the fracture.

Conflict of interest None.

\section{References}

1. Protzman RR, Burkhalter WE (1976) Femoral-neck fractures in young adults. J Bone Joint Surg Am 58:689-695

2. Dedrick DK, Mackenzie JR, Burney RE (1986) Complications of femoral neck fracture in young adults. J Trauma 26:932-937

3. Kofoed H (1982) Femoral neck fractures in young adults. Injury 14:146-150

4. Upadhyay A, Jain P, Mishra P et al (2004) Delayed internal fixation of fractures of the neck of the femur in young adults: a prospective, randomised study comparing closed and open reduction. J Bone Joint Surg Br 86:1035-1040
5. Barnes R, Brown JT, Garden RS et al (1976) Subcapital fractures of the femur. J Bone Joint Surg Br 58B:2-24

6. Alberts KA, Jervaeus J (1990) Factors predisposing to healing complications after internal fixation of femoral neck fracture. A stepwise logistic regression analysis. Clin Orthop 257:129-133

7. Alho A, Benterud JG, Ronningen H et al (1992) Prediction of disturbed healing in femoral neck fracture. Radiographic analysis of 149 cases. Acta Orthop Scand 63:639-644

8. Estrada LS, Volgas DA, Stannard JP et al (2002) Fixation failure in femoral neck fractures. Clin Orthop 399:110-118

9. Weinrobe M, Stankewich CJ, Mueller B et al (1998) Predicting the mechanical outcome of femoral neck fractures fixed with cancellous screws: an in vivo study. J Orthop Trauma 12:27-36

10. Garden RS (1961) Low-angle fixation in fractures of the femoral neck. J Bone Joint Surg Br 43B:647-663

11. Byrd JWT (2005) Surgical Technique: supine position. Oper Tech Orthop 15:204-217

12. Beck M, Leunig M, Clark E et al (2004) Femoroacetabular impingement as a factor in the development of non-union of the femoral neck: a report of three cases. J Orthop Trauma 18(7): $425-430$ 